\title{
TWO-LAYER PLATE MECHANICAL METAMATERIALS
}

\author{
Jonathan Bryan ${ }^{\dagger}$ Eric Lu ${ }^{\dagger}$, Keivan Davami, John Cortes, Chen Lin, Drew E. Lilley, and Igor Bargatin* \\ University of Pennsylvania, USA
}

\begin{abstract}
We present the fabrication and characterization of plates made from two ultrathin layers and capable of withstanding repeated extreme mechanical deformation. The ultrathin layers, one flat and the other corrugated, are less than $100 \mathrm{~nm}$ thick and created using atomic layer deposition, forming a joined structure similar to conventional honeycomb sandwich plates. The structures exhibit a high bending stiffness while still possessing a low area density of approximately $0.5 \mathrm{~g} / \mathrm{m}^{2}$. Finite element simulations show that the bending stiffness initially increases quadratically with the height of the plate, but saturates at very large heights. Measurements of bending stiffness using an atomic force microscope agree qualitatively with the simulations.
\end{abstract}

\section{INTRODUCTION}

Cellular solids offer low weight and unique mechanical and thermal properties, which explains their longstanding use for a variety of structural applications in the transportation and construction industries [1]. In the last decade, a novel class of cellular solids known as mechanical metamaterials has been introduced [2-3]. These solids have carefully designed and tightly controlled periodic 3D geometries, usually at the micro- or nanoscale, which lead to unique combinations of mechanical properties, allowing them to occupy new areas on the Ashby charts [4-6].

Recently, we introduced the concept of plate mechanical metamaterials [7] - cellular plates with carefully controlled periodic geometry and unique mechanical properties - as well as its initial realization in the form of freestanding corrugated plates made out of an ultrathin film. In particular, we used atomic layer deposition (ALD) and microfabrication techniques to make robust plates out of a single continuous ALD layer with $\mathrm{cm}$-scale lateral dimensions and a thickness as low as $25 \mathrm{~nm}$, creating the thinnest freestanding plates that can be picked up by hand [7]. Our continuous plates differ from "bulk mechanical metamaterials" reported by other groups (e.g., Refs. [2-6]), which form a lattice that is easily penetrated by air and are typically made using much slower fabrication techniques, such as nanoscale 3D printing [4-5] or evaporation-driven self-assembly [6].

At the macro scale, many types of periodic cellular plates are used. In particular, honeycomb lattices and sandwich structures, which consist of two face sheets attached to a periodic core, have become ubiquitous in construction, aerospace, scientific instrumentation (e.g., optical tables), and other industries that require lightweight rigid plates $[8,9]$. Sandwich structures possess a high bending stiffness and very low areal density (mass per unit area). However, macroscale sandwich plates generally cannot sustain sharp bending deformations without permanent damage [9].

Here, we report fabrication and characterization of fully suspended plate metamaterials made from two layers of nanoscale thickness, whose geometry and properties are reminiscent of honeycomb sandwich plates (Fig. 1). The two layers are offset from each other but at the same time are connected using hexagonal vertical walls, which prevent shear of the two layers with respect to one another. As a result, the two-layer plates offer much higher bending stiffness than the single-layer structures we reported earlier [7], while still possessing extremely low weight and mechanical robustness. The increase in the bending stiffness is expected, and its mechanism is similar to that used in conventional honeycomb sandwich plates. However, in contrast to sandwich composite plates, our nanoscale two-layer mechanical metamaterials can sustain extremely large deformations without fracture, fully recovering their original shape and not displaying any signs of internal damage.

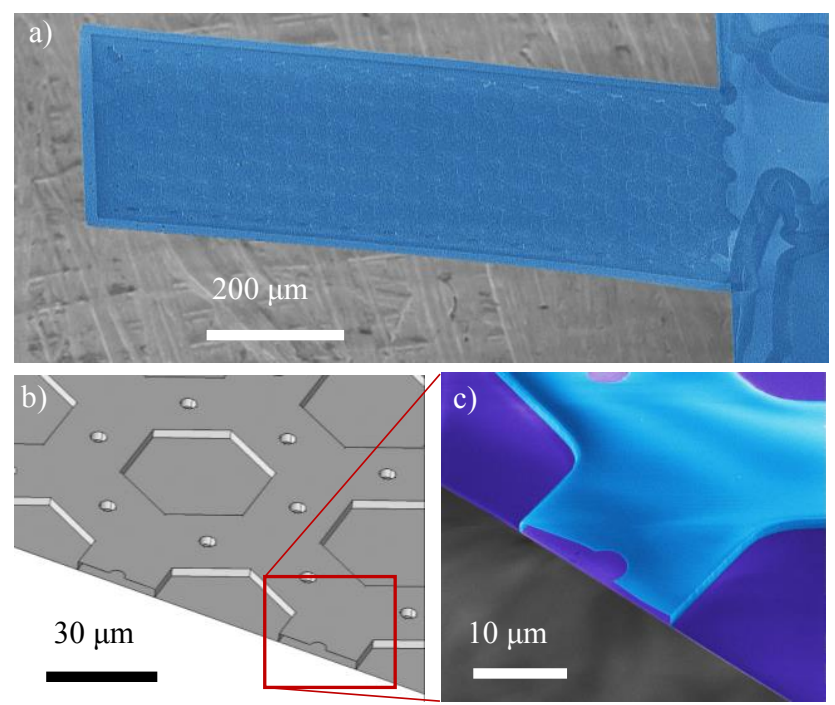

Figure 1: (a) SEM image of a 1-mm-long and 0.5-mm-wide cantilevered plate made from a two-layer mechanical metamaterial. Because the freestanding ALD layers are highly transparent in $S E M$, they are colored for clarity. (b) Schematic illustrating the periodic geometry of the two-layer plate. The bottom layer is planar and continuous while the top layer is corrugated and includes etch holes for release. (c) SEM showing the detail of the cantilever edge. The layers are approximately 60-nm-thick and are colored differently for clarity. The plate height, i.e., the spacing between the two layers, is nominally 2 microns, but in practice varies slightly in a fully released structure.

\section{FABRICATION}

The plates were fabricated from ALD aluminum oxide (alumina) as illustrated in Fig. 2. First, we deposited a 60-nm-thick planar layer of aluminum oxide $\left(\mathrm{Al}_{2} \mathrm{O}_{3}\right)$ on a cleaned double-side polished silicon wafer. The deposition was performed at $250{ }^{\circ} \mathrm{C}$ using water and trimethylaluminum precursors in the ALD tool. Next, a sacrificial layer of amorphous silicon (a-Si) was deposited at $175^{\circ} \mathrm{C}$ on the front side using plasma-enhanced chemical vapor deposition (PECVD). The thickness of the a-Si layer, which determines the height of the finished plate and the spacing between the ALD film layers, varied between 1 and 3 microns in different fabrication runs. The a-Si layer was then patterned using photolithography and anisotropic reactive ion etching (RIE) to obtain the hexagonal honeycomb pattern shown in Fig. 1b. The hexagonal honeycomb geometry was chosen because it exhibits approximately isotropic bending stiffness [1,7]. Subsequently, another 60-nm-layer of ALD alumina was deposited to form the corrugated layer. The second ALD layer was then patterned using photolithography and inductively coupled plasma (ICP) RIE to define the width and length of the cantilever as well as to open the etch holes for removing the a-Si sacrificial layer later on. Next, an approximately 500-nm-thick layer of silicon nitride was deposited 
on the back side of the wafer and patterned to serve as a hard mask for $\mathrm{KOH}$ wet-etching. The wafer was then backside-etched in $\mathrm{KOH}$, stopping $10-50 \mu \mathrm{m}$ before reaching the ALD layers. Finally, the device was suspended using isotropic $\mathrm{XeF}_{2}$ etching or isotropic $\mathrm{CF}_{4}+\mathrm{O}_{2}$ plasma $\mathrm{RIE}$, which also removed the sacrificial a-Si trapped between the ALD layers. The SEM images confirm the presence of two alumina layers and the removal of the sacrificial layer (Fig. 1c).
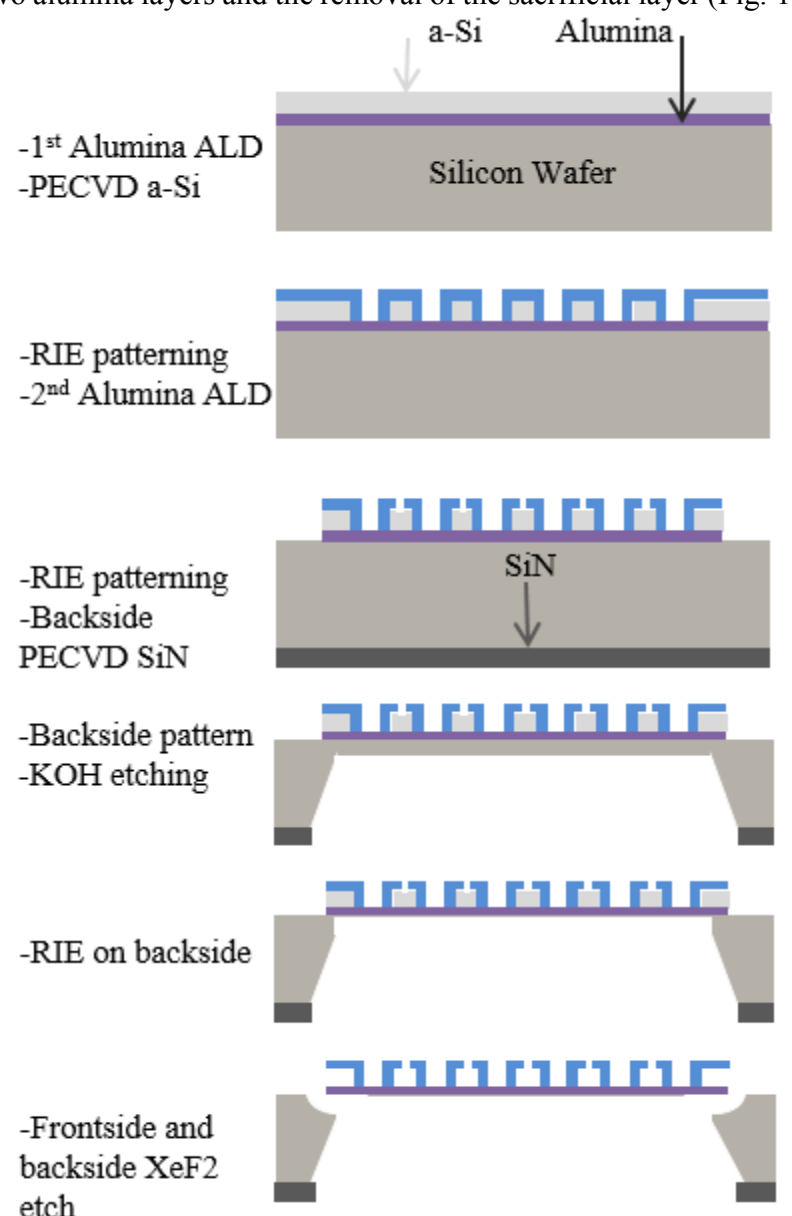

Figure 2: Schematic of the fabrication steps.

(a)

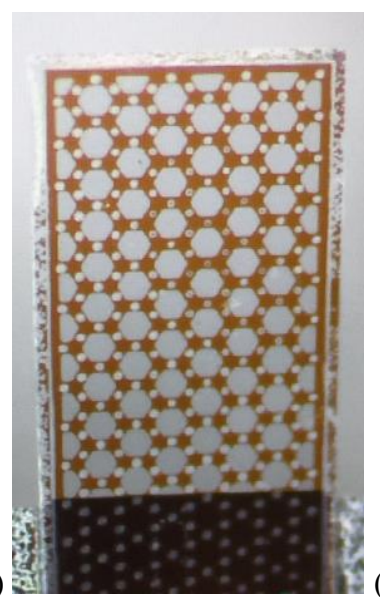

Figure 3: Photographs of partly (a) and fully (b) etched cantilevers under an optical microscope. The remaining a-Si trapped between the ALD alumina layers appears brown in the partly etched cantilever (a). In contrast, the completely etched cantilever without residual amorphous silicon (b) is completely clear (transparent).
The complete removal of the trapped $\mathrm{Si}$ is critical because the unique mechanical properties of plate mechanical metamaterials depend on the extremely low thickness of the structural ALD layers. Any residual a-Si dramatically changed the mechanical properties of the cantilevers, leading to easy fracturing during sharp bending deformations. Therefore, the etching of a-Si was continued until all evidence of a-Si disappeared in optical and scanning electron micrographs. Because the ALD alumina is highly transparent compared to a-Si, the presence of the a-Si could be easily detected in optical micrographs (Fig. 3).

\section{SIMULATIONS OF BENDING STIFFNESS}

The two-layer geometry described above is much easier to realize using MEMS fabrication processes than the exact analog of a standard honeycomb sandwich composite [8,9], which would consist of two planar layers (faces) and a corrugated honeycomb layer in between them. However, based on our finite element simulations and experiments, the stiffness of the fabricated twolayer structures is comparable to that of a full honeycomb composite.

The small-deformation response of the two-layer plate structures was studied using the shell interface of COMSOL Multiphysics (version 5.0). The layers were modeled as alumina films with isotropic linear elastic properties, a Young's modulus of $130 \mathrm{GPa}$, and a Poisson ratio of $0.22[7,10]$. Mesh convergence was achieved using triangular elements with a minimum and maximum element size of 0.005 and 10 microns respectively. Geometric parameters were set to coincide with those of the experimental cantilever. To find the spring constant of a cantilever and the corresponding bending stiffness of the plate material, one end of the cantilever was fixed while a normal, out-of-plane load was applied at the other end. Mirror symmetry conditions were applied to the edges along the cantilever's length.

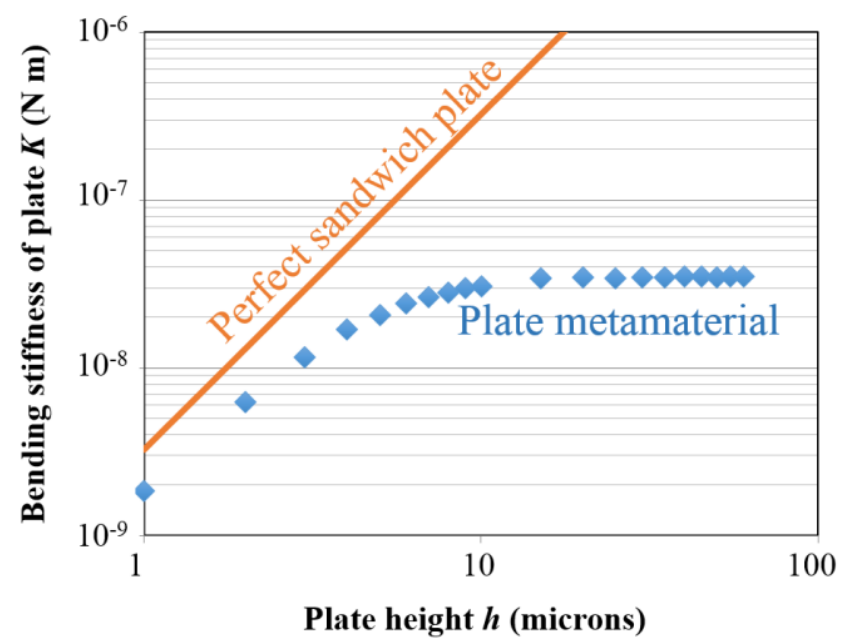

Figure 4: The symbols show the simulated effective bending stiffness $K_{\mathrm{eff}}$ of the two-layer plates as a function of the plate height. For comparison, the solid line shows the bending stiffness of a perfect sandwich plate of the same height $(h)$ made from the films of the same thickness $(t): K_{\text {sandwich }}=Y t h^{2} / 2$, where $Y=130 \mathrm{GPa}$ is the Young's modulus of the ALD aluminum oxide.

Figure 4 shows the bending stiffness $K_{\text {eff }}$ of two-layer plate metamaterial as predicted by the finite element simulations. We note that the spring constant $k$ of the cantilever with a width $W_{\text {cant }}$ and length $L_{\text {cant }}$ is related to the plate bending stiffness by the formula $k=3 W_{\text {cant }} K_{\text {eff }} / L_{\text {cant }}^{3}$. The simulations show that for low heights, the bending stiffness of the fabricated two-layer plates increases 
quadratically with the plate height. It also remains approximately a factor of two lower than the bending stiffness of a perfect sandwich plate, i.e., a plate consisting of two face sheets and a corrugated layer between them that completely prevents the lateral shear of the face sheets with respect to one another. The fact that the bending stiffness is lower than that of a perfect sandwich structure is not surprising, given that the top layer of our plates is not perfect: it contains etch holes and is completely absent inside the hexagons. At heights above $\sim 10 \mu \mathrm{m}$, the bending stiffness saturates as the vertical hexagonal walls that connect the top and bottom ALD layers can no longer prevent the shear of the top layer with respect to the bottom layer.

Still, even for the relatively low heights reported here $(1-3 \mu \mathrm{m})$, the bending stiffness of this two-layer plate mechanical metamaterial is many orders of magnitude higher than that of singlelayer plate mechanical metamaterials reported earlier. From Fig. 3, the effective bending stiffness is predicted to be between $K_{\text {eff }} \approx$ $2 \times 10^{-9} \mathrm{~N} \cdot \mathrm{m}$ for $h=1 \mu \mathrm{m}$ and $\approx 10^{-8} \mathrm{~N} \cdot \mathrm{m}$ for $h=3 \mu \mathrm{m}$. In contrast, single-layer plate metamaterials with similar in-plane geometry and the same $60-\mathrm{nm}$ thickness of the ALD film have bending stiffness of only $3 \times 10^{-11} \mathrm{~N} \cdot \mathrm{m}$. By adding a second layer, we therefore achieved 2-3 orders of magnitude improvement in the bending stiffness of the plate material while the mass per unit area is less than doubled. The areal density of the two layer-structures can be easily calculated from the geometry and the density of the amorphous aluminum oxide $\left(\rho=4000 \mathrm{~kg} / \mathrm{m}^{3}\right)$ and is approximately 0.5 gram per square meter.

\section{MEASUREMENTS OF BENDING STIFFNESS}

To validate the predictions of the finite element simulations, we determined the spring constants of the cantilevers that were made from two-layer plate mechanical metamaterials (shown in Fig. 1 and 2). In particular, force-displacement curves were obtained at the tip of each cantilever using an Asylum atomic force microscope (AFM). Given the relatively low spring constants of the two-layer plates, we used soft AFM cantilever tips with a spring constant of $k_{\text {AFM tip }} \approx 0.01 \mathrm{~N} / \mathrm{m}$ and $0.26 \mathrm{~N} / \mathrm{m}$, which were determined using the thermal noise method. By modeling the plate as a spring in series with the AFM cantilever, the slope of the force displacement curve corresponds to the effective spring constant $K_{\text {total }}$, which satisfies $k_{\text {total }}^{-1}=k_{\text {AFM tip }}^{-1}+k_{\text {plate }}^{-1}$.

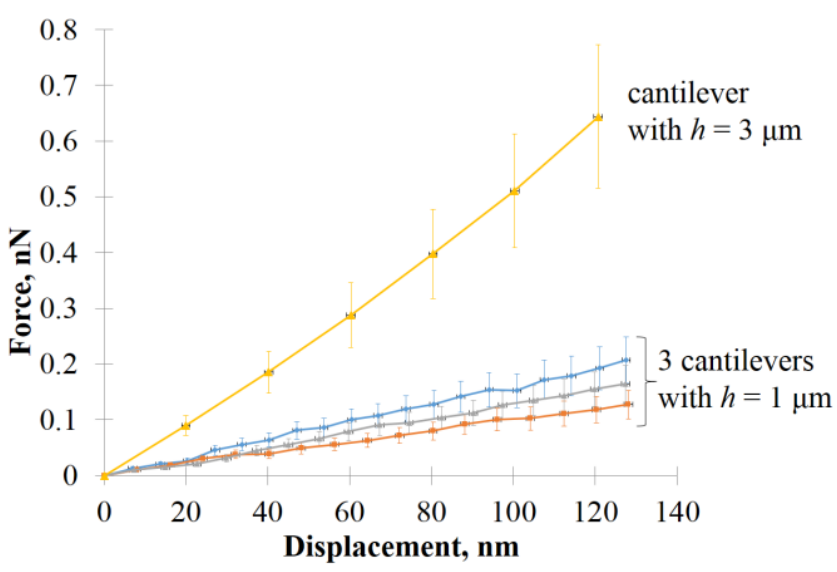

Figure 4: Force-displacement curves of two-layer plate cantilevers obtained at the tip of each cantilever using an atomic force microscope. The 1- $\mu$ m-tall cantilevers were measured using an

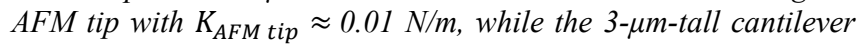
was measured using an AFM tip with $K_{A F M ~ t i p} \approx 0.26 \mathrm{~N} / \mathrm{m}$.
Using this equation, we determined that the spring constants were $1.5 \pm 0.3 \mathrm{mN} / \mathrm{m}$ and $5.4 \pm 1.1 \mathrm{mN} / \mathrm{m}$ for the $1-\mu \mathrm{m}$-tall and 3 $\mu \mathrm{m}$-tall cantilevers, respectively. Using the cantilever width of $W_{\text {cant }}=0.45 \mathrm{~mm}$ and the length of $L_{\text {cant }}=1 \mathrm{~mm}$, we could also determine the effective bending stiffness of the plate material: $K_{\text {eff }}=k L_{\text {cant }}^{3} / 3 W_{\text {cant }}$, which yielded bending stiffness of $(1.1 \pm 0.2) \times 10^{-10} \mathrm{~N} \cdot \mathrm{m}$ and $(4 \pm 1) \times 10^{-10} \mathrm{~N} \cdot \mathrm{m}$ for the $1-\mu \mathrm{m}$-tall and $3-\mu \mathrm{m}$-tall cantilevers, respectively. These measured bending stiffnesses are approximately a factor of two lower than those predicted by finite element simulations (Fig. 4), which could be attributed to the fact that the actual spacing between the top and bottom layers is lower in experimental fully released devices than the nominal design values (Fig. 1). Despite this discrepancy, the predicted trend of increasing bending stiffness with increasing plate height is fully supported by the experiments. In the future, we plan to fabricate structures with even larger heights to test the prediction that the bending stiffness saturates at large heights.

\section{RECOVERY FROM EXTREME BENDING}

The most surprising property of our two-layer plates is their ability to sustain sharp bending without deformation. We observed the mechanical robustness of the two-layer plates using a micromanipulator probe while imaging the device in an FEI Strata focused ion beam (FIB) tool. Similar to the single-layer plates reported earlier [7], the two-layer plates showed a remarkable ability to recover from several extreme deformations without any apparent fracture or permanent deformation (Fig. 5). This is strikingly different from the behavior of macroscale multilayer composites, such as honeycomb sandwich plates, which typically fracture, delaminate, or irreversibly buckle after such sharp bending $[8,9]$.

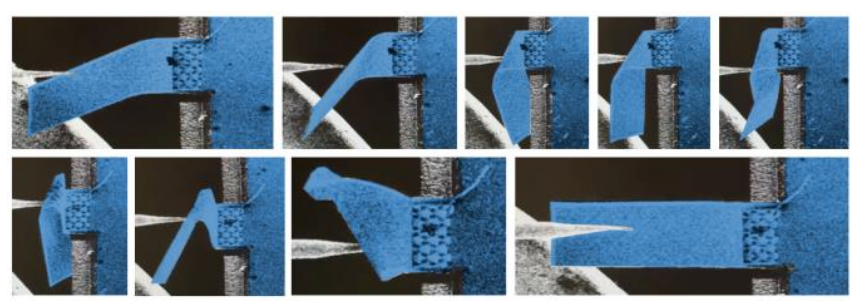

Figure 5: Sequential screenshots from a live video feed of an FIB

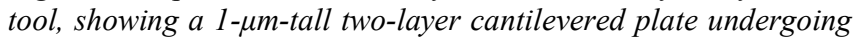
extreme bending deformations and then recovering its original shape.

The exact nature of this observed robustness is not fully clear at this point. As discussed in our previous publication [7], sandwich plates that undergo very sharp bends are expected to develop strains of the order of 1 unless the structure of plate changes. Real sandwich plates undergoing sharp bending or folding therefore either fracture completely or irreversibly change their structure $[8,9]$. For example, the face sheets of a conventional sandwich composite can delaminate from the hexagonal webbing that connects the two face sheets. Alternatively, the hexagonal webbing can be crumpled by irreversible buckling. In any of these cases, the sandwich plate typically remains permanently deformed and weakened in the area that underwent sharp bending or folding.

Yet, high resolution scanning electron micrographs do not reveal any evidence of irreversible changes after extreme bending of our two-layer plates (Fig. 6). While the plates clearly change their internal structure by locally buckling the vertical walls during sharp bending, the deformations appear to be fully elastic, reversing after the external force is removed. While the exact form of this elastic local buckling deformation needs to be investigated further, we note that similar recoverable deformations have been observed in "bulk 
mechanical metamaterials" and, in particular, nanolattices made from ultrathin ALD aluminum oxide [5].
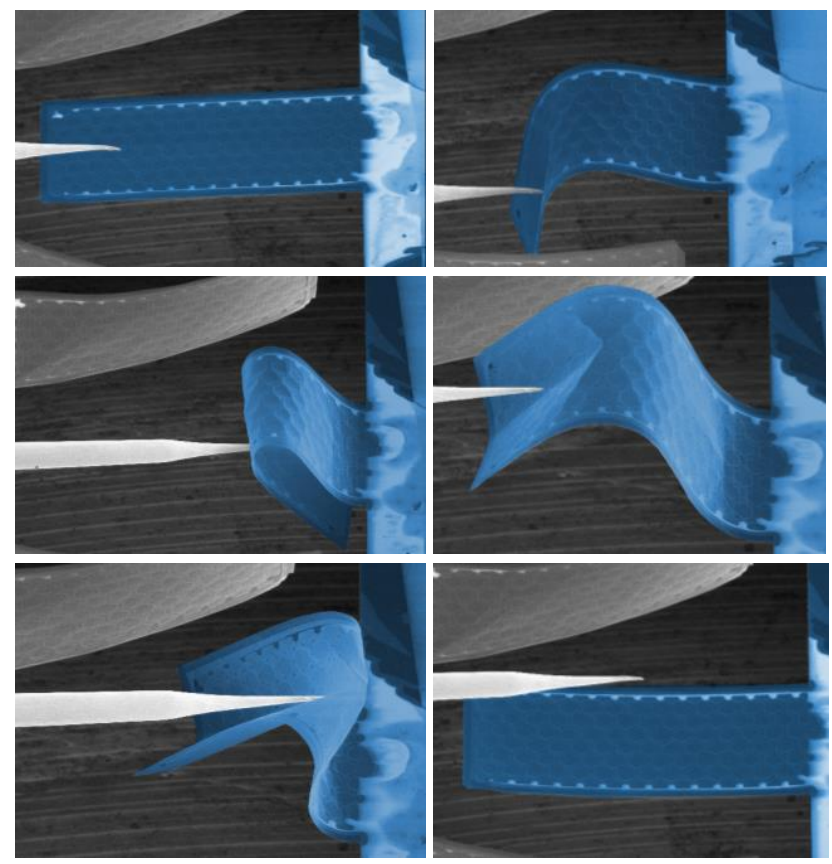

Figure 6: Sequential scanning electron micrographs illustrating a 2- $\mu$-tall two-layer cantilevered plate undergoing extreme bending deformations and then recovering its original shape. After the recovery, the plate did not display any signs of damage even in highresolution SEM images of the areas where sharp folding occurred.

\section{CONCLUSION}

We have demonstrated shape-recovering, flat, ultrathin, continuous plate mechanical metamaterials made out of two ALD layers. Relative to previously reported single-layer plates [7], the bending stiffness of two-layer plates is increased by 2-3 orders of magnitude and is, in fact, within a factor of two of that of an ideal sandwich plate. At the same time, the two-layer plates still feature ultralow areal density ( $\sim 0.5$ gram per square meter) and can be made using a relatively high-throughput fabrication method that can be easily scaled to $\mathrm{cm}$-scale plates. Like the single-layer plates reported earlier, the two-layer plate mechanical metamaterials can pop back elastically to their original position after extremely sharp bending deformations without any apparent damage. This is in striking contrast to macroscopic sandwich composites, which typically fracture, delaminate, or irreversibly buckle after sharp bending. The observed robustness and the apparent lack of irreversible deformation are likely due to reversible buckling of ultrathin structures [5].

Ultralow weight, mechanical robustness, thermal insulation, as well as chemical and thermal stability of alumina make two-layer plate metamaterials attractive for numerous applications, including structural elements in flying microrobots, high-temperature thermal insulation in energy converters, testing of nanoscale strength enhancement, new types of optical and acoustic metamaterials, as well as ultra-lightweight hollow MEMS sensors and resonators.

\section{ACKNOWLEDGMENTS}

The authors thank the dedicated staff of the Quattrone Nanofabrication Facility at the University of Pennsylvania for their help with fabrication. The use of the University of Pennsylvania's Nano/Bio Interface Center (NBIC) instrumentation is acknowledged. This work was supported, in part, by the School of
Engineering and Applied Science at the University of Pennsylvania and a seed grant from the Center of Excellence for Materials Research and Innovation (CEMRI), NSF grant DMR11-20901.

\section{REFERENCES}

[1] J.L. Gibson, M.F. Ashby, "Cellular Solids: Structure and Properties" (Cambridge University Press, 2001).

[2] T.A. Schaedler, A.J. Jacobsen, A. Torrents, A. E. Sorensen, J. Lian, J.R. Greer, L. Valdevit, W.B. Carter, "Ultralight metallic microlattices". Science 334, 962-965 (2011).

[3] J.H. Lee, J. P. Singer, E. L. Thomas, Micro-/Nanostructured Mechanical Metamaterials. Adv. Mater. 24, 4782-4810 (2012).

[4] X. Zheng, H. Lee, T.H. Weisgraber, M. Shusteff, J. DeOtte, E.B. Duoss, J.D. Kuntz, M.M. Biener, Q. Ge, J.A. Jackson, S.O. Kucheyev, N.X. Fang, C.M. Spadaccini "Ultralight, ultrastiff mechanical metamaterials", Science 344, 1373-1377 (2014).

[5] L.R. Meza, S. Das, J.R. Greer, "Strong, lightweight, and recoverable three-dimensional ceramic nanolattices", Science 345, 1322-1326 (2014).

[6] J.H. Pikul, Z. Dai, X. Yu, H. Zhang, T. Kim, P.V. Braun, W.P. King, "Micromechanical devices with controllable stiffness fabricated from regular 3D porous materials", Journal of Micromechanics and Microengineering 24, 105006 (2014).

[7] K. Davami, L. Zhao, E. Lu, J. Cortes, C. Lin, D. Lilley, P.K. Purohit, and I. Bargatin, "Ultralight shape-recovering plate mechanical metamaterials," Nature Communications, 6, 10019 (2015).

[8] J.R. Vinson, "The Behavior of Sandwich Structures of Isotropic and Composite Materials" (CRC Press, 1999).

[9] T.N. Bitzer, "Honeycomb Technology: Materials, Design, Manufacturing, Applications and Testing" (Springer, 1997).

[10] O.M.E. Ylivaara, X. Liu, L. Kilpi, J. Lyytinen, D. Schneider, M. Laitinen, J. Julin, S. Ali, S. Sintonen, M. Berdova, E. Haimi, "Aluminum oxide from trimethylaluminum and water by atomic layer deposition: The temperature dependence of residual stress, elastic modulus, hardness and adhesion", Thin Solid Films 552, 124-135 (2014).

$\dagger$ The first two authors have contributed equally to this work.

\section{CONTACT}

*I. Bargatin, tel: +1-215-746-4887; bargatin@seas.upenn.edu 